arteriovenous malformation drains into branches of PVs or sometimes directly into the LA or inferior vena cava. They usually are embedded to lung parenchyma.

In addition to causing shunt resulting in dyspnea, volume overload, and cyanosis, PAVMs also cause the loss of filter function of lungs and thus may allow development of stroke and cerebral abscess. The classic triad of dyspnea, cyanosis, and clubbing is present in only $10 \%$ of cases. ${ }^{1}$

In our case, the lesion was producing high-output right-to-left shunt and causing severe cyanosis. The aneurysm had a single large feeding artery (afferent vessel) and no efferent vessel. Interestingly, it was in continuity with the LA with an orifice as big as the aneurysm itself. In our knowledge no similar case has been reported in literature.

Traditional options for the definitive management of PAVM include embolization and surgery. Ligation, local excision, segmentectomy, lobectomy, or pneumonectomy have been performed, ${ }^{1,2}$ although most of the time parenchyme-saving operations are preferred.

It was obvious that embolization would be inappropriate for this case not only because leaving a residual aneurysm would result in a high risk of rupture but also because of the unsuitable anatomy of the feeding artery. During preoperative evaluation we considered 2 possible approaches for surgical treatment of this PAVM. Instead of median sternotomy and repair through the LA with cardiopulmonary bypass and cardiac arrest, we used simple ligation and resection through thoracotomy, which allowed better exposure, a shorter operation, and faster recovery. We believe the origin of this sac would be one of the following: an aneurysmal PAVM with draining vessel was absorbed in time and united widely to the LA, or a distal PA aneurysm ruptured and united to the LA. Unusual features like extraparenchymal location of the lesion, absence of an efferent vessel, unity of aneurysmal sac with the LA, and the absence of associated etiologic factors make this lesion interesting. Nonetheless, prompt surgical treatment with a simple approach relieved the severe symptoms and prevented a potential fatal rupture.

In conclusion, although PAVMs are rare, they should be included in any differential diagnosis of worsening dyspnea, cyanosis, stroke, or hemoptysis. Their presence should be considered especially in patients with cyanosis whom no evidence of intracardiac shunts in echocardiography. Prompt workup and intervention can prevent fatal complications like rupture and lead to dramatic improvement in symptoms.

\section{References}

1. Gossage JR, Kanj G. Pulmonary arteriovenous malformations. A state of the art review. Am J Respir Crit Care Med. 1998;158:643-61.

2. Khurshid I, Downie Gh. Pulmonary arteriovenous malformation. Postgrad Med J. 2002;78:191-7.

3. Duncan BW, Desai S. Pulmonary arteriovenous malformations after cavopulmonary anastomosis. Ann Thorac Surg. 2003;76:1759-66.

\title{
Use of the endoclamp device in a patient with a native mitral valve endocarditis and a previous arch replacement with a modified Griepp technique
}

\author{
Siamak Mohammadi, MD, Pierre Voisine, MD, Patrick Mathieu, MD, and François Dagenais, MD, Québec City, Canada
}

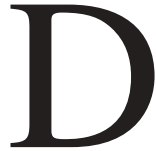

evelopment of new catheters and cannulas has allowed accessing the heart through short incisions. Use of this technology may also be applied for difficult reoperative situations. We herein report the use of the endoclamp in a patient with a previous root and arch replacement requiring mitral surgery for a native mitral endocarditis.

\footnotetext{
From the Department of Cardiac Surgery, Laval Hospital, Québec City, Québec Canada.

Received for publication Nov 25, 2005; accepted for publication Jan 3, 2006.

Address for reprints: Dr François Dagenais, Department of Cardiac Surgery, Laval Hospital, 2725 Chemin Ste-Foy, Québec, Canada, G1V 4G5

(E-mail: francois.dagenais@chg.ulaval.ca).

J Thorac Cardiovasc Surg 2006;131:1188-90

$0022-5223 / \$ 32.00$

Copyright $\odot 2006$ by The American Association for Thoracic Surgery doi:10.1016/j.jtcvs.2006.01.007
}

\section{Clinical Summary}

A 58-year-old male was admitted with asthenia, weight loss, and fever. His past medical history was positive for hypertension, diabetes, peripheral vascular disease (aortobifemoral graft). The patient had a root replacement with a Bjork-Shiley conduit in 1982 for a type A dissection. The patient also had an arch replacement using an isolated graft to the arch vessels (modified Greipp technique) and an "elephant trunk" procedure in 2001 with a right axillary artery cannulation. The patient also required a replacement of his thoracoabdominal aorta in May 2002.

Transthoracic echocardiography revealed a moderate mitral regurgitation and a $3.5-\mathrm{cm}$ vegetation on the posterior leaflet. No vegetation was documented on the Bjork-Shiley aortic prosthesis. Blood cultures grew Staphylococcus epidermidis. Owing to his poor overall condition, the patient was treated with vancomycin and rifampycin although the embolic risk was present. Absence of dacron graft infection was suggested by a normal gallium scan and magnetic resonance imaging. 


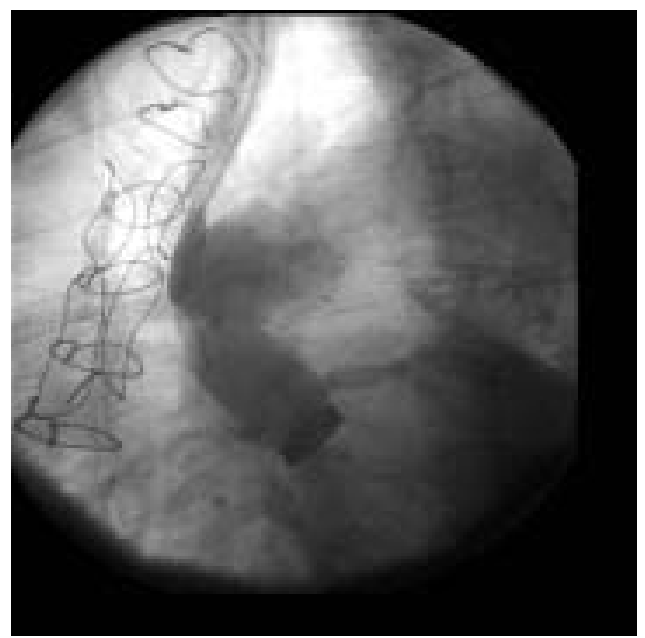

Figure 1. Sketch showing the endoclamp position and the arterial perfusion through the right limb of the aortobifemoral graft and the right axillary artery.

Following a 4-week antibiotic course, the patient's overall condition improved and a reoperation was considered. Repeat echocardiography showed similar findings. An approach through the right chest was selected owing to the multiple grafts. To optimize exposure of the mitral valve with an aortic prosthesis, decompression of the aortic root was considered to be preferable. To initiate aortic crossclamping and root decompression, an endoclamp was inserted in the Y port of the arterial cannula (Cardiovations, Somerville, NJ) of the right limb of the aortobifemoral graft (Figure 1). Owing to the limited space to fully inflate the endoclamp between the arch graft and the aortic valve (Figure 2), cerebral perfusion was ensured by recannulating the right axillary artery. A pulmonary vent (Cardiovations) was inserted through the right jugular vein. The endoclamp was inflated under transesophageal echocardiography guidance just above the aortic valve thus covering the arch vessel graft. Cardioplegia and root decompression were conducted through the endoclamp ports. The left atrium was opened and adequate exposure of the mitral valve obtained. The vegetation was localized on the posterior leaflet. The mitral valve was replaced with a mechanical prosthesis. Total aortic crossclamp and cardiopulmonary bypass times were 88 and 113 minutes, respectively. Postoperatively, the patient developed a right pleural effusion necessitating 2 pleurodeses. At 8-month follow-up, the patient is well and free of infection.

\section{Discussion}

Mideline chest reentry for mitral surgery with previous proximal aortic surgery can be hazardous. In such circumstances, the right anterolateral thoracotomy has been shown to be safe and effective. ${ }^{1,2}$

During the last decade, minimal access of the mitral valve has been advocated either with or without the port-access platform. Excellent results have been reported with minimal access mitral procedures even in previously operated patients. ${ }^{3}$ The present case illustrates the use of the port-access platform in a complex case with multiple previous thoracic aortic surgeries. Because a redo sternotomy

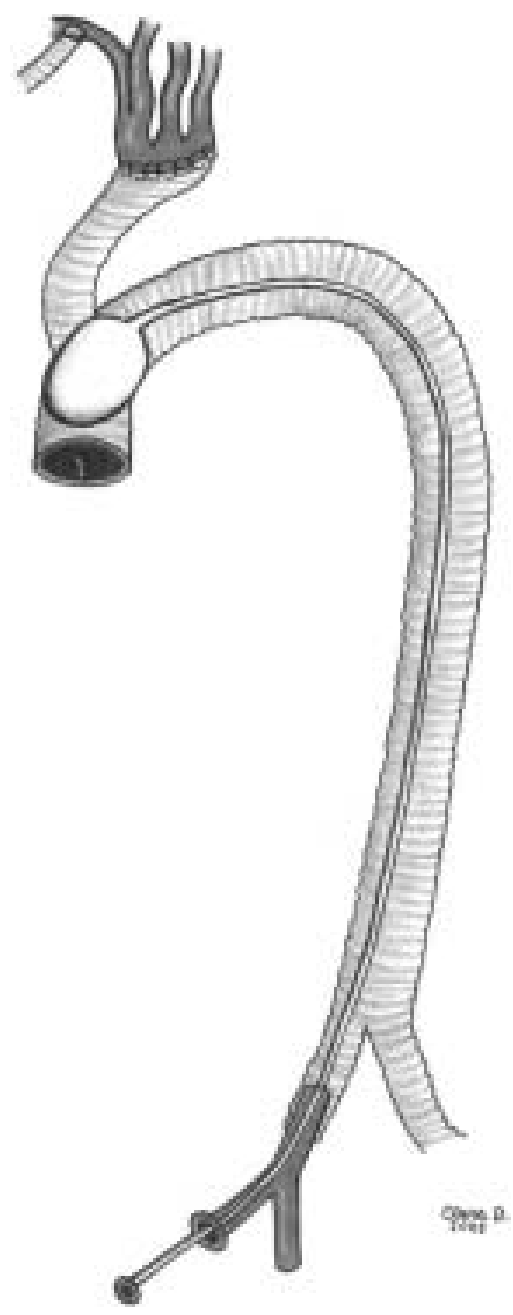

Figure 2. Aortogram demonstrating the narrow space between the arch graft and the aortic valve.

was judged prohibitive, access through the untouched right chest, as opposed to the left chest, seemed the easiest and safest approach. Although mitral surgery through the right chest under ventricular fibrillation or on the beating heart has been reported, ${ }^{4}$ the presence of a mechanical valve with a pressurized root may limit exposure of the mitral valve. To obviate this problem, insertion of an endoclamp through the femoral cannula allowed arresting the heart and venting the aortic root. However, the distance between the aortic prosthesis and the takeoff of the arch graft did not permit safe inflation of the endoclamp. To ensure adequate arch vessel perfusion, the right axillary artery was reused. We have previously reported the feasibility and the safety of recannulating the right axillary artery. ${ }^{5}$ Other possible options could have been to cannulate the right brachial artery or the left axillary artery.

In summary, we report a unique case of mitral valve surgery with previous multiple aortic surgeries including an arch replacement with a modified Griepp technique. Use of the port-access technology enabled us to arrest the heart and vent the aortic root. 
In case of an isolated graft to the arch vessel, one should ensure sufficient space to inflate the endoclamp without compromising cerebral perfusion. If uncertain, one should consider cannulating the brachial or axillary artery to perfuse the brain during the endoclamp inflation.

\section{References}

1. Galla JD, McCullough JN, Grippe RB. Aortic arch replacement for dissection: A comparative atlas. Op Tech Thorac Cardiovasc Surg. 1999;4:58-76.
2. Holman WL, Goldberg SP, Early LJ, McGiffil DC, Kirklin JK, Cho DH, et al. Right thoracotomy for mitral reoperation: analysis of the technique and outcome. Ann Thorac Surg. 2000;70:1970-3.

3. Burfeind WR, Glower DD, Davis RD, Landolfo KP, Lowe JE, Wolfe WG. Mitral surgery after prior cardiac operation: port-access versus sternotomy or thoracotomy. Ann Thorac Surg. 2002;74:S1323-5.

4. Ghosh S, Jutley RS, Wraighte P, Shajar M, Nailk SK. Beating-heart mitral valve surgery in patients with poor left ventricular function. J Heart Valve Dis. 2004;13:622-7.

5. Shetty R, Voisine P, Mathieu P, Dagenais F. Recannulation of the right axillary artery for complex aortic surgeries. Tex Heart Inst J. 2005; 32:194-7.

\section{Ventricular assist device use for the treatment of acute viral myocarditis}

Veli K. Topkara, MD, Nicholas C. Dang, MD, Fabio Barili, MD, Timothy P. Martens, MD, Isaac George, MD, Faisal H. Cheema, MD, Hasmet Bardakci, MD, Ali Vefa Ozcan, MD, and Yoshifumi Naka, MD, PhD, New York, NY

A cute viral myocarditis is a rare condition and associated with high mortality due to the rapid development of heart failure. Ventricular assist devices (VADs) have become a life-saving approach for patients with acute viral myocarditis who are otherwise refractory to the aggressive medical therapy. ${ }^{1,2}$ We reviewed our institutional experience to evaluate the use of VADs as a treatment option for viral myocarditis.

\section{Patients and Methods}

From January 1995 to March 2005, 11 patients underwent VAD implantation (left VAD [LVAD] in 10, biventricular assist device [BIVAD] in 1) for acute viral myocarditis at Columbia-Presbyterian Medical Center. Patients were evaluated with regards to demographics, presenting symptoms, histological manifestations, and electrocardiographic findings. Outcome variables included bridgeto-transplantation rate and long-term survival. Data was collected by retrospective chart review.

From the Columbia University College of Physicians and Surgeons, Department of Cardiothoracic Surgery, New York, NY.

Received for publication July 26, 2005; accepted for publication Aug 3, 2005.

Address for reprints: Yoshifumi Naka, MD, PhD, Herbert Irving Assistant Professor of Surgery, Columbia University, College of Physicians and Surgeons, Director, Mechanical Circulatory Support Program, New YorkPresbyterian Hospital, Columbia University Medical Center, 177 Fort Washington Avenue, Milstein Hospital 7GN-435, New York, NY 10032 (E-mail: yn33@columbia.edu).

J Thorac Cardiovasc Surg 2006;131:1190-1

$0022-5223 / \$ 32.00$

Copyright $\odot 2006$ by The American Association for Thoracic Surgery doi:10.1016/j.jtcvs.2005.08.073

\section{Results}

The mean age of the population was $33.8 \pm 14.2$ years. None of the patients had a previous history of cardiac disease. Prodromal symptoms preceding the onset of myocarditis included flulike symptoms, chest pain, syncope, and varicella syndrome (Table 1). All patients were transferred from outside institutions with diagnoses of decompensated heart failure complicating acute viral myocarditis. The diagnosis of viral myocarditis was primarily based on clinical presentation and histologic findings and supported by viral serology and culture. Viral pathogens were isolated only in 6 of the 11 patients (Table 2). Histologic evidence of acute myocarditis using the Dallas Criteria was present in 8 patients (72.7\%); only cellular infiltrate was found in the remaining patients. ${ }^{3}$ At the time of admission to our center, 4 patients were temporarily supported by either intra-aortic balloon pump $(\mathrm{n}=3)$ or extracorporeal membrane oxygenation (ECMO; $\mathrm{n}=1$ ). All patients had electrocardiographic abnormalities including sinus tachycardia, diffuse nonspecific repolarization abnormalities, and bundle branch block.

LVAD was used as the sole support system in 8 patients with left ventricular failure. BIVAD was temporarily implanted in 2 patients with biventricular failure and was switched to LVAD after recovery of right ventricular function. One patient was bridged to transplant using BIVAD alone without the necessity for LVAD.

There were no operative mortalities $(0.0 \%)$. Nine patients had postoperative complications including acute renal failure, right heart failure, reoperation for bleeding, pericardiocentesis for cardiac tamponade, and sepsis (Table 1). The mean duration of VAD support was $58.4 \pm 91.7$ days (range 5-324 days). Four patients (36.4\%) died in-hospital on VAD support with a mean survival of $12 \pm 6$ postoperative days. Causes of death for these patients included right heart failure $(\mathrm{n}=2)$, sepsis $(\mathrm{n}=1)$, and multiorgan failure $(\mathrm{n}=1)$. The mean preoperative LVAD score was significantly higher in patients who died compared with those who survived $(6.3 \pm 1.3$ vs $3.5 \pm 1.9, P=.034) .{ }^{4}$ Of the 7 surviving 